\title{
Successfull Management of a Life Threatening Cerebellar Haemorrhage Following Spine Surgery - A Case Report -
}

\author{
Johan Pallud ${ }^{*}$, Hayat Belaïd ${ }^{\dagger}$, Sorin Aldea ${ }^{\dagger}$ \\ *Sainte-Anne Hospital Center, Department of Neurosurgery, University René Descartes, Paris, France, \\ ${ }^{\dagger}$ Pitié-Salpêtrière Hospital, Department of Neurosurgery, Paris, France, \\ ${ }^{\dagger}$ Foch Hospital, Department of Neurosurgery, Suresnes, France
}

\begin{abstract}
Cerebellar haemorrhages are rare life-threatening complications following spine surgery that present challenges for their diagnostic and their therapeutic management. Their patho-physiology remains unclear.

We report a case of a life-threatening cerebellar haemorrhage secondary to an occult dural tear following a planned L5-S1 laminectomy. The patient was treated with emergent external ventriculostomy following by a posterior fossa decompressive craniectomy. Cerebellar haemorrhages have to be suspected systematically when unexpected neurological signs occur after spine surgery since their rapid management lead to favourable outcomes. The present imaging findings allow us proposing that cerebellar haemorrhages result primarily from superior cerebellar venous stretching and tearing, and that cerebellar infarction and swelling occur secondarily.
\end{abstract}

Key Words: Cerebellar haemorrhage, Dural tear, Spine surgery

\section{Introduction}

Spine surgery is associated with a wide range of complications, including dural tears that lead to cerebrospinal fluid (CSF) leakages ${ }^{1,2}$. Cerebellar haemorrhages $(\mathrm{CH})$ are extremely rare and dramatic complications following spine surgery that seem to result from peroperative and/or postoperative CSF leakages. We report a case of a life threatening $\mathrm{CH}$ after lumbar spine surgery associated with an occult dural tear requiring emergent neurosurgical management. We discuss the possible mechanisms underlying such a condition and highlight the need to perform systematically a cranial computerized tomography (CT-scan) when unexpected neurological signs occur after spine surgery.

\section{Case Report}

A 73-year-old woman, without past medical history, was found to have a symptomatic degenerative spondylolisthesis at L5-S1 associated with spinal canal stenosis. The patient underwent a planned L5-S1 laminectomy and fusion with pedicle screws in prone position using a posterior approach in an outside institution (day 0). No dural tear or CSF leak was identified preoperatively and a percutaneous drain was placed. The patient had no arterial hypertension during the perioperative period and the drain, opened under gravity, drained an abundant and clear serosanguinous fluid. At day 3 , the drain was obturated and the patient was allowed to stand and walk. She then developed severe headaches and

Received Dec 1, 2008; 1st revised Jan 12, 2009; 2nd revised Jan 24, 2009; accepted Feb 2, 2009

Corresponding author: Johan Pallud, MD

Department of Neurosurgery, Sainte-Anne Hospital

1 rue Cabanis 75014 Paris, France

Tel: +33-1-4565-8487, Fax: +33-1-4077-8167, E-mail: johanpallud@hotmail.com 
confusion, and her clinical status suddenly deteriorated with a loss of consciousness. Emergent CT-scan demonstrated an acute cerebellar haemorrhage in the superior folia of the cerebellar hemispheres, an obstructive hydrocephalus and a diffuse pneumocephalus (Fig. 1). The patient was referred emergently to our institution with a Glasgow Coma Scale at 7 requiring intubation, and no focal neurological signs. Emergent external ventriculostomy was performed and drain was removed (day 3). The CSF pressure, approximated during surgery was about 15 to $18 \mathrm{~cm}$ of water. Postoperatively, the patient was transferred to intensive care. The ventricular catheter was placed with an opening pressure of $15 \mathrm{~cm}$ of water, and antiedema treatment was administered (4 mg intravenous dexamethasone every $6 \mathrm{~h}, 100 \mathrm{mg} 20 \%$ mannitol every $6 \mathrm{~h}$ ). The level of consciousness did not improve and a 48-hour postoperative CT-scan demonstrated an increased edema of the posterior fossa associated with upward and downward herniations of the cerebellum (day 5). A posterior fossa decompressive craniectomy with a large duraplasty was then performed. At day 7, the level of consciousness improved but clear fluid began leaking from the lumbar incision. Surgical exploration identified a 8-mm dural tear at the caudal aspect of the previous laminectomy. The patient fully recovered consciousness, and was neurologically intact. Both incisions healed, and the external ventriculostomy was removed at day 10 . Control CT-scan, performed one week later, demonstrated a complete resorption of the haemorrhage and a regression of posterior fossa oedema and hydrocephalus (Fig. 2). The patient was then discharged with a normal neurological examination at day 17. At 6 months of follow-up, the patient had a normal neurological examination.

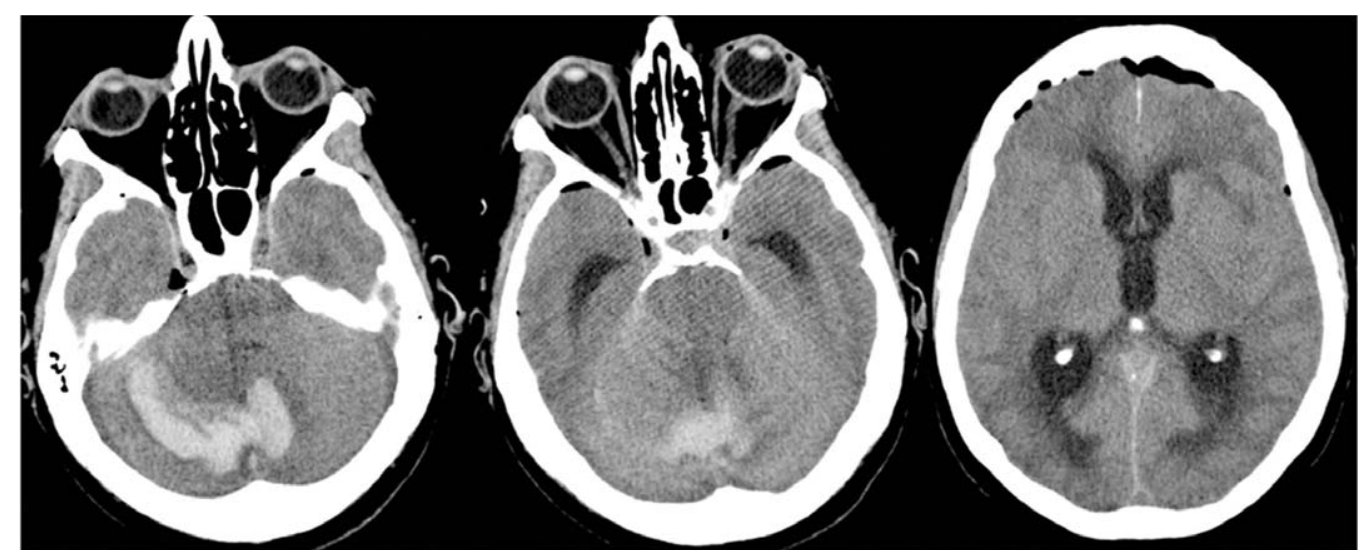

Fig. 1. Preoperative cranial non contrast-enhanced CT-scan demonstrating: 1) an acute cerebellar haemorrhage located in the superior folia of the hemispheres; 2) a compression of the fourth ventricle; 3 ) an acute obstructive hydrocephalus; 4) a pneumocephalus in the subdural spaces.

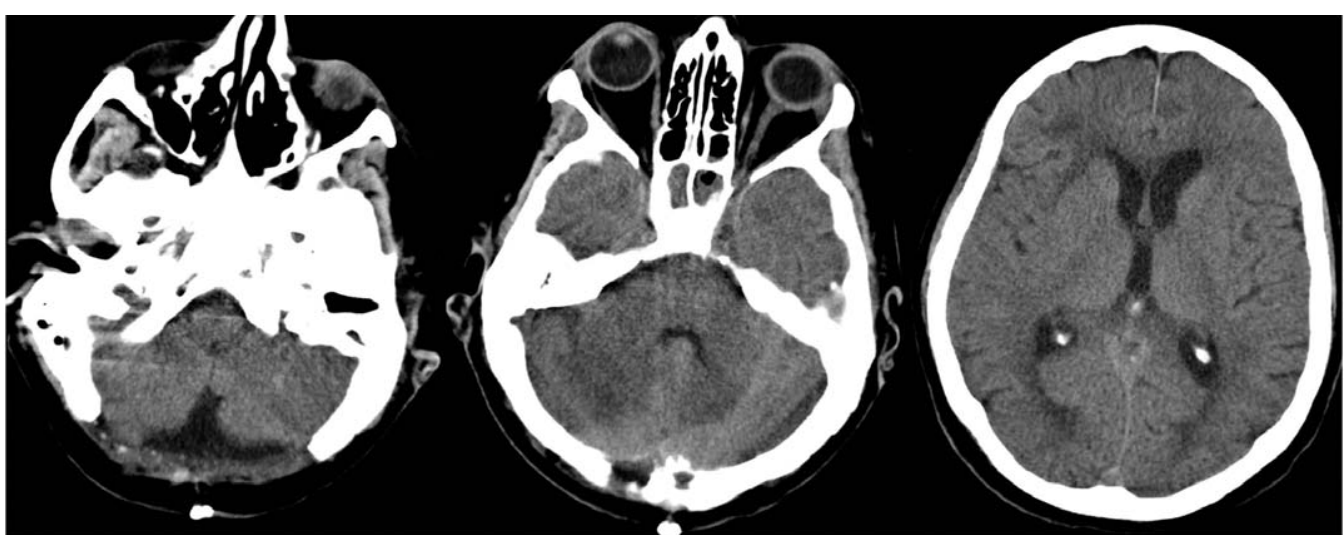

Fig. 2. Postoperative cranial non contrast-enhanced CT-scan demonstrating: 1) a complete resorption of the cerebellar haemorrhage; 2) a regression of posterior fossa swelling; 3 ) a visible fourth ventricle associated with a regression of the hydrocephalus. 


\section{Discussion}

Cerebellar haemorrhages are rare and dramatic complications following spine surgery and few cases have been published, since the first description ${ }^{3}$. In all of the documented cases, $\mathrm{CH}$ have occurred in patients with dural tear and CSF leak, whether occult or not, and the onset of neurological symptoms has been reported more than ten hours after surgery, as in the present one ${ }^{2,4}$.

It is thus admitted that peroperative and/or postoperative CSF losses, leading to intracranial hypotension, represent the main contributing factor in $\mathrm{CH}^{25,6}$. In the present case, two facts support the main role of intracranial hypotension in the setting of $\mathrm{CH}$ : 1) neurological symptoms appeared when the patient was allowed to stand up and walk, while the drains were still opened and; 2) peroperatively, during ventriculostomy, the observed CSF pressure was very low. Of note, no other aetiology, such as the use of anticoagulants, high arterial blood pressure and underlying intracranial vascular abnormalities, could explain such a complication in the present case and no correlations were found in literature between $\mathrm{CH}$ and age, sex, pathology operated or type of surgery performed ${ }^{7.8}$. The time of $\mathrm{CH}$ was in the range of previous reported cases (16-120 hours, median $16)^{7,8}$.

The exact patho-physiology of $\mathrm{CH}$ is however still controversial. If a venous origin is widely admitted, the exact mechanisms remains discussed. It has been suggested that a transient stretching and occlusion of superior cerebellar veins, resulting from downward cerebellar displacement in a situation of intracranial hypotension, may lead to cerebellar haemorrhagic infarction ${ }^{6.9}$. It has also been suggested that cerebellar sag, can directly cause a tearing and bleeding of superior cerebellar veins ${ }^{6}$. The mechanism of venous infarction was debated due to the lack of typical imaging findings $s^{6}$. In the present case, the first CTscan showed an acute cerebellar haemorrhage and surrounding edema. Diffuse cerebellar swelling occurred on the second CT-scan, concomitantly to the clinical deterioration. We can thus hypothesize that $\mathrm{CH}$ results primarily from superior cerebellar venous stretching and tearing, and that cerebellar infarction and swelling occur secondarily. We can also hypothesize that both mechanisms may co-exist.

Therefore, as a practical consequence, we recommend suspecting systematically $\mathrm{CH}$ when unexpected neurological signs occur after spine surgery, since rapid diagnosis and management lead to a favourable outcome of this lifethreatening complication.

\section{REFERENCES}

1. Bloch J, Regli L: Brain stem and cerebellar dysfunction after lumbar spinal fluid drainage: case report. J Neurol Neurosurg Psychiatry 2003; 74: 992-994.

2. Karaeminogullari O, Atalay B, Sahin O, et al: Remote cerebellar hemorrhage after a spinal surgery complicated by dural tear: case report and literature review. Neurosurgery 2005; 57: E215.

3. Chadduck WM: Cerebellar hemorrhage complicating cervical laminectomy. Neurosurgery 1981; 9: 185-189.

4. Nakazawa K, Yamamoto M, Murai K, et al: Delayed emergence from anesthesia resulting from cerebellar hemorrhage during cervical spine surgery. Anesth Analg 2005; 100: 1470-1471.

5. Andrews RT, Koci TM: Cerebellar herniation and infarction as a complication of an occult postoperative lumbar dural defect. AJNR Am J Neuroradiol 1995; 16: 13121315 .

6. Friedman JA, Ecker RD, Piepgras DG, Duke DA: Cerebellar hemorrhage after spinal surgery: report of two cases and literature review. Neurosurgery 2002; 50: 1361-1363.

7. Konya D, Ozgen S, Pamir MN: Cerebellar hemorrhage after spinal surgery: case report and review of the literature. Eur Spine J 2006; 15: 95-99.

8. Thomas G, Jayaram H, Cudlip S, Powell M: Supratentorial and infratentorial intraparenchymal hemorrhage secondary to intracranial CSF hypotension following spinal surgery. Spine (Phila Pa 1976) 2002; 27: E410-412.

9. Gobel F, Heidecke V, Hube R, et al: Cerebellar hemorrhage as an early complication of pinal operations: 2 . case reports and review of the literature. Z Orthop Ihre Grenzgeb 1999; 137: 371-375. 